\title{
A Dual Process Perspective on Congruent and Incongruent Placements as an Advertising Tactic: An Abstract
}

\author{
Claas Christian Germelmann, Jean-Luc Herrmann, Mathieu Kacha, \\ Peter R. Darke, and Jessica Weigelnée Schapfl
}

\begin{abstract}
While advertising planners often seek matching media content, some research suggests ad-medium incongruence can be more effective. We analyze the effects from a dual-process viewpoint and find that ad-medium incongruence can evoke a negative deliberative process, involving persuasion knowledge (pk), which is different from the standard fluency explanation. Experiment 1 shows that under incidental exposure, consumers focus their attention on incongruence, but not congruence. Experiment 2 confirms that incongruence leads to conscious suspicion about manipulative intent. Experiment 3 yields supporting process evidence by showing pk moderates skepticism toward the incongruent media tactic. Managerial implications for advertisers are discussed.
\end{abstract}

C. C. Germelmann $(\bowtie) \cdot$ J. Weigelnée Schapfl

University of Bayreuth, Bayreuth, Germany

e-mail: c.c.germelmann@uni-bayreuth.de; jessica.schapfl@web.de

J.-L. Herrmann · M. Kacha

Université de Lorraine, Metz, France

e-mail: jean-luc.herrmann@univ-lorraine.fr; mathieu.kacha@univ-lorraine.fr

P. R. Darke

York University, Toronto, Canada

e-mail: pdarke@schulich.yorku.ca 